\title{
Antithrombotic therapy after coronary artery stenting in atrial fibrillation: dual therapy encompassing NOAC plus P2Y12 inhibitor is ready for prime time!
}

\author{
Andreas Goette \\ Department of Cardiology and Intensive Care Medicine, St. Vincenz Hospital, Paderborn, Germany \\ Correspondence to: Prof. Dr. Andreas Goette, MD. Medizinische Klinik II, St. Vincenz-Krankenhaus, Am Busdorf 2, 33098 Paderborn, Germany. \\ Email: andreas.goette@vincenz.de. \\ Provenance: This is an invited article commissioned by the Section Editor Dr. Yiyin Zhang (Master of Oncology, Fudan University Shanghai Cancer \\ Center, Fudan University, Shanghai, China). \\ Comment on: Lopes RD, Heizer G, Aronson R, et al. Antithrombotic Therapy after Acute Coronary Syndrome or PCI in Atrial Fibrillation. N Engl J \\ Med 2019;380:1509-24.
}

Submitted Nov 18, 2019. Accepted for publication Nov 29, 2019.

doi: $10.21037 / \mathrm{atm} .2019 .12 .33$

View this article at: http://dx.doi.org/10.21037/atm.2019.12.33

Non-vitamin k antagonist oral anticoagulants (NOACs) are drugs of choice for the majority of patients with atrial fibrillation (AF) to prevent cerebral strokes (1). The risk for stroke in $\mathrm{AF}$ patients is determined by concomitant clinical factors, which are summarized in the CHA2DS2-Vasc risk score (2). Of note, in many patients AF appears to be the consequence of an atrial cardiomyopathy, which triggers the occurrence of $\mathrm{AF}$ and induces a thrombogenic endocardial remodeling process (Figure 1). The endocardial remodeling encompasses overexpression of several adhesion molecules, which may cause clot formation at the endocardial surface, and in particular in the left atrial appendage (2-6). Many AF patients will develop coronary artery stenoses, which require stent implantation (PCI). Thus, dual antiplatelet therapy (aspirin and P2Y12 inhibitors) is necessary to prevent clot formation and thrombosis of implanted stents (7-10). The main question is, what is the best antithrombotic therapy in $\mathrm{AF}$ patients requiring PCI? Dual antithrombotic regime (DAT) or triple antithrombotic regime (TAT)?

The AUGUSTUS trial was designed to assess the effect of apixaban versus warfarin in AF patients after an acute coronary syndrome (ACS) or PCI (11). The AUGUSTUS trial had a two-by-two factorial design. AF patients with an ACS or PCI were randomized to receive apixaban or a vitamin $\mathrm{K}$ antagonist and to receive aspirin or placebo. Main endpoint of this study was defined as major or clinically relevant nonmajor bleeding. Secondary endpoints were death or hospitalization and a composite of ischemic events. Lopez et al. included a total of 4,614 patients (11). Main endpoint occurred in $10.5 \%$ in the apixaban arm, as compared with $14.7 \%$ in the VKA arm (hazard ratio, $0.69 ; 95 \%$ CI, 0.58 to $0.81 ; \mathrm{P}<0.001$ ), and in $16.1 \%$ of the aspirin treated patients, as compared with $9.0 \%$ on placebo (hazard ratio, $1.89 ; 95 \% \mathrm{CI}, 1.59$ to $2.24 ; \mathrm{P}<0.001$ ). The apixaban treated patients had a lower incidence of death or hospitalization compared to the VKM arm (11). The aspirin group of the trial showed similar rates of death or hospitalization and of ischemic events compared to placebo. Thus, AF patients with ACS or PCI treated with a P2Y12 inhibitor, apixaban, without aspirin, resulted in less bleeding and fewer hospitalizations without significant differences in the incidence of ischemic events compared to the other groups studied (11).

The results of the trial are of importance and will influence clinical practice. However, the Augustus trial has some limitations. The trial randomized patients 6.6 days after the coronary event. Thus the first weeks of antithrombotic therapy was not covered in the trial. Furthermore, not all patient received PCI. Therefore, AUGUSTUS analyzed a heterogenous group of AF patients. Follow up in AUGUSTUS was rather short (6 months) and not in clear accordance to current guidelines, which recommend dual therapy in ACS patients for 12 months. In addition, triple therapy was applied for 


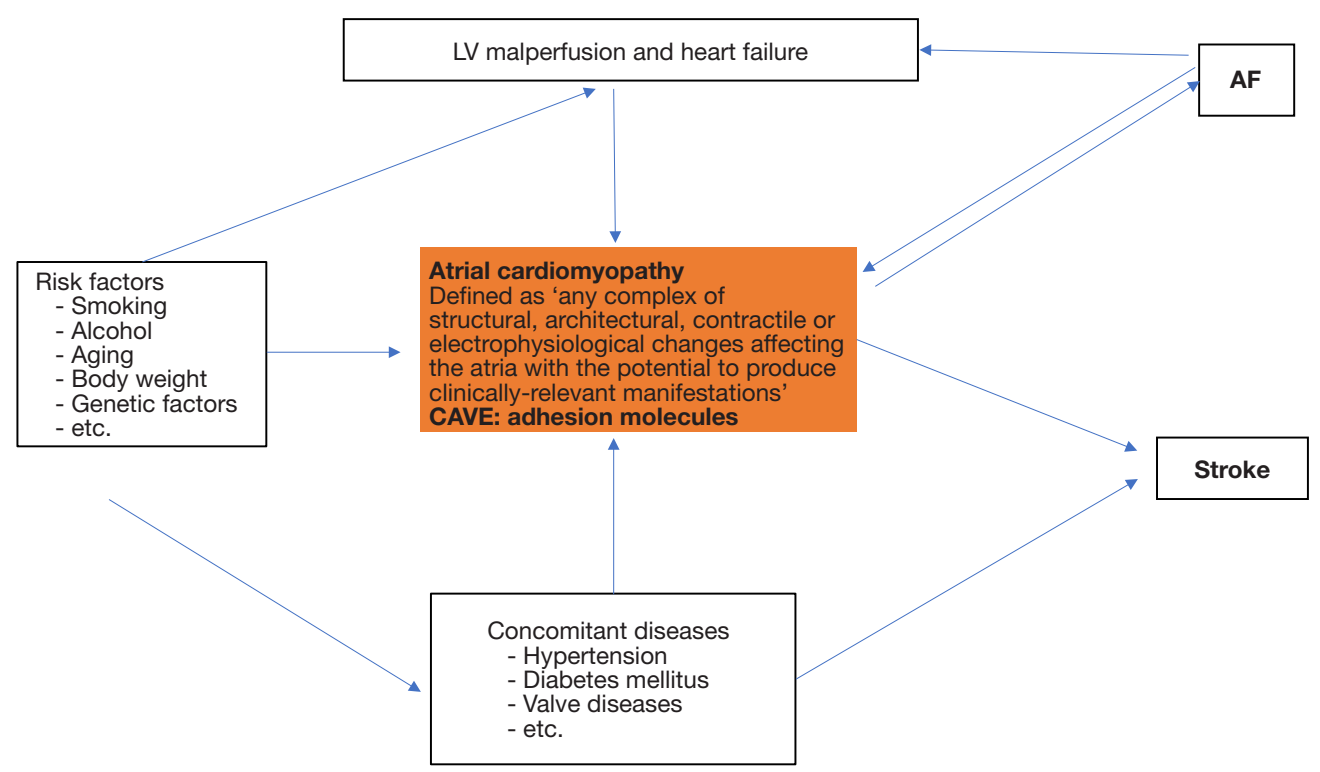

Figure 1 Impact of atrial cardiomyopathy on atrial thrombogenesis in patients with and without AF. In most patients with concomitant risk factors, AF appears to be an epiphenomenon, which characterizes the presence of an atrial cardiomyopathy. The induced endocardial alterations, in particular overexpression of adhesion molecules, cause thrombogenesis in the left atrial appendage. AF, atrial fibrillation.

6 months, which appears to be aggressive in the light of the RE-DUAL trial or ENTRUST AF PCI. A recent meta-analysis has analyzed all four trials, which assessed antithrombotic therapy in AF patients with coronary artery diseases with and without stenting (12). This analysis clearly showed that dual therapy consisting of a NOAC plus a p2Y12 inhibitors is safer than triple therapy encompassing a VKA, aspirin and a P2Y12 inhibitor. Of note, it was also shown that the use of aspirin is of importance in the early phase after stenting to protect the stent against thrombotic events. This first full meta-analysis confirmed previous findings on the advantage of DAT over TAT with respect to bleeding complications (12). However, this analysis showed a significant reduction of intracranial haemorrhage with DAT as compared to TAT. This finding is consistent with prior NOAC studies focusing on AF but non-PCI patients $(12,13)$. Interestingly, there was high heterogeneity across the four AF PCI trials with respect to bleeding endpoints (12). Another clinically relevant finding from the meta-analysis is the increased rates of cardiac ischemia and stent thrombosis. This aspect is relevant and reinforces the notion that the upfront selection between TAT or DAT and/or the optimal timing for aspirin discontinuation after invention or ACS should be individualised (12). The mechanisms through which early aspirin discontinuation exposes patients to higher ischemic risks remain speculative.
Thus, triple therapy is of importance in all $\mathrm{AF}$ patients after stenting for some weeks to prevent stent thrombosis $(12,13)$. In contrast, this strategy is not necessary in AF patients with ACS who are medically managed as demonstrated by the AUGUSTUS trial. After 14 days of triple therapy all AF patients might be reduced to dual therapy to reduce the cumulative bleeding risk. After 6 months (in stabile coronary artery disease) or after 12 months (ACS) dual therapy should be terminated and NOAC therapy should be continued as monotherapy (Figure 2A,B).

The ENTRUST AF PCI trial suggests that bleeding rates after PCI differ between radial and femoral access (8). Thus, the periprocedural antithrombotic regime might address this aspect. In most centers, which use the femoral rout, oral anticoagulants including NOACs are paused 24 hours prior to the procedure. Re-initiation of therapy might start about 24 hours after the procedure to reduce bleeding from the femoral access site. This temporary pause of NOACs might not be necessary if the radial route is used for PCI (Figure 2C).

Overall, the AUGUSTUS trial in addition to the other three AF PCI trials adds substantial clinical information to the field (8-11). Further substudies are warranted to assess differences between various subgroups of AF patients like ACS and stable CAD (CCS). A latest report from AUGUSTUS suggest than aspirin therapy should be given 
A

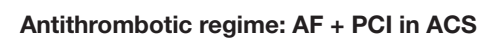

3

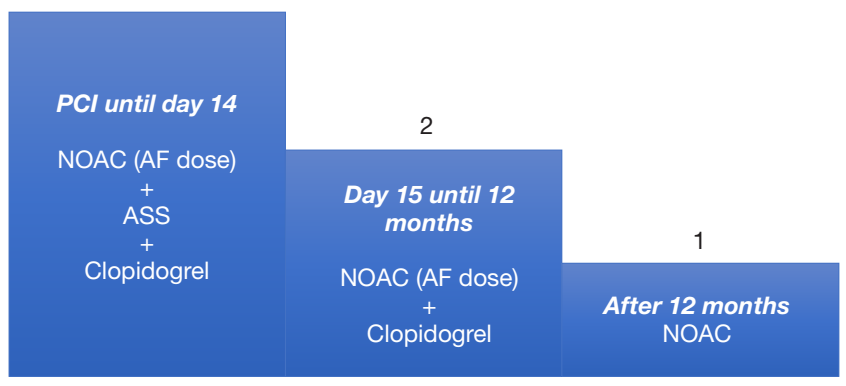

B

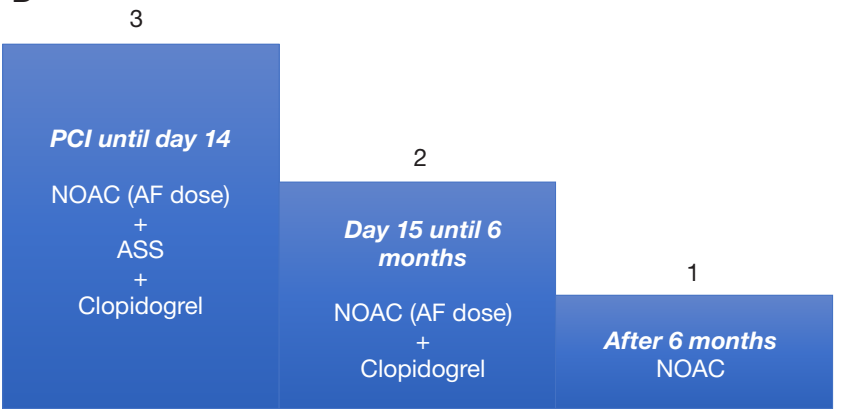

C

Periinterventional Anticoagulation \& Antiplatelet Therapy after $\mathrm{PCl}$ in AF Patients

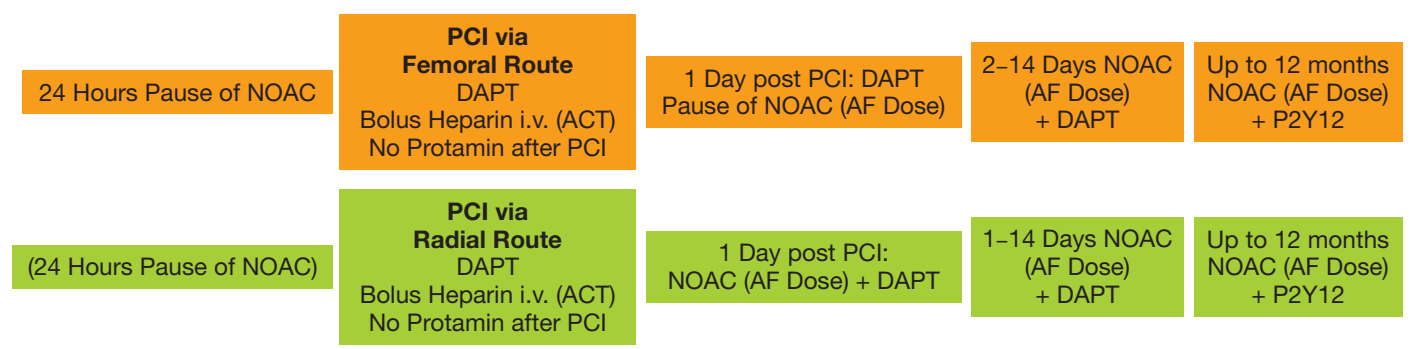

Figure 2 (A) Summary of the antithrombotic regime in patients with AF and ACS during follow up; (B) summary of the antithrombotic regime in patients with $\mathrm{AF}$ and chronic coronary syndrome (CCS, stable coronary artery disease); (C) periprocedural regime of antithrombotic therapy in AF patients undergoing PCI. AF, atrial fibrillation; ACS, acute coronary syndrome; NOAC, non-VKA-antagonist; PCI, percutaneous coronary intervention with stent implantation; DAPT, dual antiplatelet therapy (ASS + P2Y12 inhibitor); ACT, activated clotting time to guide heparin dose; $\mathrm{AF}$ dose, NOAC dose per lable to prevent stroke in atrial fibrillation patients.

for at least one week in patients with low risk of stent thrombosis and up to 30 days in patients at high risk (14). Therefore, the suggested time period of 14 days of aspirin therapy (Figures $2 A, B$ ) appears as a compromise since aspirin induces an irreversible inhibition of platelet function, and therefore, platelets are inhibited up to 28 days after 14 days of continuous aspirin therapy.

\section{Acknowledgments}

This work was supported by Research grant from the JosefFreitag-Stiftung Paderborn and Deutsche Herzstiftung.

\section{Footnote}

Conflicts of Interest: Speaker fees from Abbott, Astra Zeneca, Bayer Health Care, Berlin Chemie, Biotronik, Boehringer Ingelheim, BMS/Pfizer, Boston Scientific, Daiichi-Sankyo, Medtronic, and Omeicos.

Ethical Statement: The author is accountable for all aspects of the work in ensuring that questions related to the accuracy or integrity of any part of the work are appropriately investigated and resolved.

\section{References}

1. Ruff CT, Giugliano RP, Braunwald E, et al. Comparison of the efficacy and safety of new oral anticoagulants with warfarin in patients with atrial fibrillation: a meta-analysis of randomised trials. Lancet 2014;383:955-62.

2. Goette A, Kalman JM, Aguinaga L, et al. EHRA/ HRS/APHRS/SOLAECE expert consensus on atrial cardiomyopathies: definition, characterization, and clinical implication. Europace 2016;18:1455-90.

3. Bukowska A, Lendeckel U, Krohn A, et al. Atrial fibrillation down-regulates renal neutral endopeptidase expression and induces profibrotic pathways in the kidney. Europace 2008;10:1212-7.

4. Goette A, Jentsch-Ullrich K, Lendeckel U, et al. Effect of atrial fibrillation on hematopoietic progenitor cells: a novel pathophysiological role of the atrial natriuretic peptide? Circulation 2003;108:2446-9.

5. Merino JL, Lip GYH, Heidbuchel H, et al. Determinants 
of left atrium thrombi in scheduled cardioversion: an ENSURE-AF study analysis. Europace 2019;21:1633-8.

6. Bukowska A, Zacharias I, Weinert S, et al. Coagulation factor $\mathrm{Xa}$ induces an inflammatory signalling by activation of protease-activated receptors in human atrial tissue. Eur J Pharmacol 2013;718:114-23.

7. Vranckx P, Lewalter T, Valgimigli M, et al. Evaluation of the safety and efficacy of an edoxaban-based antithrombotic regimen in patients with atrial fibrillation following successful percutaneous coronary intervention (PCI) with stent placement: Rationale and design of the ENTRUST-AF PCI trial. Am Heart J 2018;196:105-12

8. Vranckx P, Valgimigli M, Eckardt L, et al. Edoxaban-based versus vitamin $\mathrm{K}$ antagonist-based antithrombotic regimen after successful coronary stenting in patients with atrial fibrillation (ENTRUST-AF PCI): a randomised, openlabel, phase 3b trial. Lancet 2019;394:1335-43.

9. Cannon CP, Batt DL, Oldgren J, et al. Dual Antithrombotic Therapy with Dabigatran after PCI in Atrial Fibrillation. N Engl J Med 2017;377:1513-24.

10. Gibson CM, Mehran R, Bode C, et al. Prevention of

Cite this article as: Goette A. Antithrombotic therapy after coronary artery stenting in atrial fibrillation: dual therapy encompassing NOAC plus P2Y12 inhibitor is ready for prime time! Ann Transl Med 2019;7(Suppl 8):S270. doi: 10.21037/ atm.2019.12.33 bleeding in patients with atrial fibrillation undergoing PCI. N Engl J Med 2016;375:2423-34.

11. Lopes RD, Heizer G, Aronson R, et al. Antithrombotic Therapy after Acute Coronary Syndrome or PCI in Atrial Fibrillation. N Engl J Med 2019;380:1509-24.

12. Gargiulo G, Goette A, Tijssen J, et al. Safety and efficacy outcomes of double vs. triple antithrombotic therapy in patients with atrial fibrillation following percutaneous coronary intervention: a systematic review and metaanalysis of non-vitamin $\mathrm{K}$ antagonist oral anticoagulantbased randomized clinical trials. Eur Heart J 2019. [Epub ahead of print].

13. Lopes RD, Hong H, Harskamp RE, et al. Safety and Efficacy of Antithrombotic Strategies in Patients With Atrial Fibrillation Undergoing Percutaneous Coronary Intervention: A Network Meta-analysis of Randomized Controlled Trials. JAMA Cardiol 2019. [Epub ahead of print].

14. Lopes RD, Leonardi S, Wojdyla DM, et al. Stent Thrombosis in Patients with Atrial Fibrillation Undergoing Coronary Stenting in the AUGUSTUS Trial. Circulation 2019. [Epub ahead of print]. 